Complementary

Medicine Research

Practice $\mid$ Methods | Perspectives

\title{
Evidence-Based Methods of Complementary Medicine: A Claim for Their Transfer into Practical Use in Chronic Diseases
}

\author{
Karin Kraft \\ Department of Naturopathy, University Medicine Rostock, Rostock, Germany
}

This first issue of Complementary Medicine Research has a focus on the therapy of chronic diseases. It includes 5 clinical studies on chronic diseases with high impact on the health system. The results were achieved with various scientific approaches. Though preliminary, they are predominantly promising and hopefully stimulating further research as well as practical transfer:

- In a randomised waitlist-controlled study in patients with chronic non-specific neck pain, a series of 10 cupping massages appeared to be effective in reducing pain and increasing function and quality of life [1].

- In a study on Chinese herbal medicine, the survival benefits of herb pairs were calculated by cluster analyses, association rules, and survival evaluation in 95 patients with esophageal cancer. From 176 prescriptions, which included 178 herbs, 1 promising pair could be identified, which improved overall survival [2].

- In a non-interventional study in patients with sleep disorders (with or without functional cardiovascular disorders), the Pittsburgh Sleep Quality Index was significantly reduced by $60 \%$ after 3-6 months of treatment with an anthroposophic remedy composed of 3 medicinal drugs [3].

- In a randomized controlled clinical trial, 14 sessions of electromyogram biofeedback in addition to usual care did not improve the health status in 36 elderly patients with long-standing fibromyalgia. The study was however severely underpowered, which could explain the negative results [4].

- In an uncontrolled, open-label multicenter trial including 100 patients with prolonged or chronic fatigue symptoms, a therapy with a dry ethanolic extract of Rhodiola rosea was studied. The patients showed statistically significant improvement after 8 weeks of treatment [5].

High quality clinical studies on methods of complementary medicine allow their inclusion into e.g. German medical guidelines, which are a valuable source of information not only for physicians, but increasingly also for patients with chronic diseases, who urgently need and seek independent information, especially on complementary medicine.

Indeed, independent information on complementary medicine is not easily available for patients. This problem was addressed by a recent study, which analyzed the contents of Canadian complementary-and-alternative-medicine (CAM) clinic websites for advertisement on tests and treatments of allergies and asthma. The following methods were recommended for allergies: Chiropractic adjustment, ionic foot bath detoxification, electroacu-

\section{KARGER}

() 2017 S. Karger GmbH, Freiburg

Fax +497614520714 
puncture according to Voll, muscle response testing, intravenous hydrogen peroxide, Bowen therapy, acupuncture/acupressure, $\mathrm{pH}$ testing, biofeedback analysis, fish oil consumption, ozone therapy, nutritional supplements, electrodermal testing, homeopathic remedies, sublingual immunotherapy, drinking broths regularly, regular detoxification, subcutaneous immunotherapy, neural therapy, probiotics, immunoglobulin-G testing, vitamin and mineral injection, immunoglobulin-E testing, bioresonance therapy, and intravenous vitamin C. $32 \%$ of the treatment claims for allergy were accompanied by additional explicit claims as to treatment efficacy [6].

These claims raise ethical issues because evidence in support of many of these tests and treatments is lacking. Only very few of these procedures are demonstrably clinically effective, revealing a transfer gap in complementary medicine. Also, several proposed treatments are potentially harmful, such as intravenous hydrogen peroxide and spinal manipulation. An important negative effect of using invalid and inaccurate allergy testing is the likelihood that such testing will lead to alterations and exclusions in diets. These risks are especially serious for children. Some of these tests and treatments can result in higher healthcare resource usage, which leads to financial exploitation of the patient and the public health system.

The authors reason from these results that there is a 'need for a legal and/or policy response in order to prevent potential harm and financial exploitation. One strategy would be to increase the scope of advertising regulations and enforcement, and to explore the potential of applying evidence-based standards and restricting practitioners' ability to offer unproven tests and treatments' [6].

Moreover, also German websites of CAM practitioners offer unproven or even harmful tests and therapies, and claim them to be effective. Beyond legal frameworks restricting such advertisements, I urgently recommend the implementation of the 'WHO Traditional Medicine Strategy: 2014-2023' [7] in Germany, following the successful policy of several other nations. This strategy paper includes the establishment and support of national research facilities on traditional medicine, an increased control of health professionals, and the allocation of independent free-of-charge information. All of us can contribute to this strategy by publishing more high-quality clinical studies on complementary medicine and implementing them into practice in order to provide evidencebased complementary medicine.

\section{References}

1 Saha FJ, Schumann S, Cramer H, et al: The effects of cupping massage in patients with chronic neck pain - a randomised controlled trial. Complement Med Res 2017;24: doi: 10.1159/000454872.

2 Cai Y-M, Zhu H, Niu J-X, et al: Identification of herb pairs in esophageal cancer. Complement Med Res 2017; 24: doi: 10.1159/000454699.

3 Rother C, Schnelle M: Cardiodoron ${ }^{\circledR}$ bei Patienten mit Schlafstörungen - Ergebnisse einer prospektiven Beobachtungsstudie. Complement Med Res 2017;24: doi: $10.1159 / 000452236$.
4 Baumueller E, Winkelmann A, Irnich D, Weigl M: Electromyogram biofeedback in patients with fibromyalgia: a randomized controlled trial. Complement Med Res 2017;24: doi: 10.1159/000454692.

5 Lekomtseva Y, Zhukova I, Wacker A: Rhodiola rosea in subjects with prolonged or chronic fatigue symptoms: results of an open-label clinical trial. Complement Med Res 2017;24: doi: 10.1159/000457918.
6 Murdoch B, Carr S, Caulfield T: Selling falsehoods? A cross-sectional study of Canadian naturopathy, homeopathy, chiropractic and acupuncture clinic website claims relating to allergy and asthma. BMJ Open 2016; 6:e014028.

7 World Health Organization: WHO Traditional Medicine Strategy: 2014-2023. Geneva, World Health Organization, 2013,www.who.int/medicines/publications/ traditional/trm_strategy14_23/en. 